\title{
Modelo de integración de formación profesional de la carrera de licenciatura en enfermería en la UTEQ.
}

\section{Integration model of professional training for the undergraduate degree in nursing at UTEQ.}

Lourdes María Velázquez Lores. ${ }^{1}$, Carlos Mego Cubas. ${ }^{2}$ \& Gabriel Arturo Pazmiño Solys. ${ }^{3}$

\begin{abstract}
.
DOI: $\underline{\text { https://doi.org/10.33262/concienciadigital.v3i3.1.1378 }}$

Introduction: The curricular redesign is fundamental, that is why this issue was discussed, in this case of an educational service institution such as the STATE UNIVERSITY OF QUEVEDO, among the 5 most important in the country. The idea of this proposal is the redesign of the curricular mesh of the Nursing Undergraduate Career with the insertion of the subjects that will help the student once completed their studies to face the beginning of their professional life with practical and theoretical knowledge, demonstrating what they have learned during their student time. The methodology of this research is of the qualitative, quantitative (mixed) and descriptive model, achieving the elaboration of a feasible proposal with technical elements such as the calculation of the participant population sample collecting information to be processed later. Objective: To determine a model of integration of professional training in the Nursing Career of the State Technical University of Quevedo. Methodology. Descriptive-explanatory study that covers the methodology in the quantitative and qualitative approaches (MIXED) of research., With an analysissynthesis, documentary analysis and content of literature referring to the subject was used; Original and review articles were consulted in databases: PubMed, CINAHL, SciELO, Medline, Lilacs, BIREME, Scival and Conhrane among others, according to DeCS: Curriculum, Competence-Based Education, Nursing Education. The universe studied was the totality of full-time teachers of the academic unit, being approachable,

\footnotetext{
${ }^{1}$ Magister en enfermedades infecciosas. Aspirante al grado de Doctor en Educación PhD. Hospital Sagrados Corazones. Provincia de los Ríos. Ecuador., lulyargelia@gmail.com.

${ }^{2}$ Doctor. Universidad Cesar Vallejo. Piura. Perú., mcegoc_@hotmail.com

${ }^{3}$ Doctor en Ingeniería Industrial. PhD. Master en Gestion de Proyectos Sociales y Productivos. Gaps Representaciones., gapsrio@gmail.com
} 
$\mathrm{N}=21$ was studied in total. The criteria for selecting participants were: teaching nurses from the career staff, with more than 5 years of permanence in it. Results. The process of curricular innovation of the nursing career of the UTEQ is immersed in policies and agreements that respond to the institutional educational model; attending to the increase in coverage of tertiary or higher education, social transformations and paradigm shifts in health. With the curricular innovation, it was sought to configure a new university model that, without neglecting cognitive solvency and professional competence, places preferential emphasis on the training of reflective, responsible, critical, innovative and versatile subjects to guarantee universal health coverage. The main processes and strategies that were used in this curricular innovation were in accordance with the institutional policies of the universities, following the guidelines of the institutional educational models, strategic teaching objectives inserted in the strategic development plans of the universities.

Keywords: Model, integration, training, professional, nursing

\section{Resumen}

Introducción: El rediseño curricular es fundamental, es por esto que se trató este tema, en este caso de una institución de servicio educativo como es la UNIVERSIDAD ESTATAL DE QUEVEDO, entre las 5 más importantes del país. La idea de esta propuesta es el rediseño de la malla curricular de la Carrera de Licenciatura Enfermería con la inserción de las asignaturas que ayudaran al estudiante una vez concluido sus estudios afrontar con conocimientos prácticos y teórico el inicio de su vida profesional demostrando lo aprendido durante su tiempo de estudiante . La metodología de esta investigación es del modelo cualitativo cuantitativo (mixto) y descriptivo, logrando la elaboración de una propuesta factible con elementos técnicos como son el cálculo de la muestra poblacional participante recopilando información para luego ser procesada. Objetivo: Determinar un Modelo de integración de formación profesional en la Carrera de Enfermeria de la Universidad Tecnica Estatal de Quevedo. Metodología. Estudio descriptivo-explicativo que cobija la metodología en los enfoques cuantitativo y cualitativo (MIXTA) de investigación., con un análisis-síntesis, se usó análisis documental y de contenido de literatura referente al tema; se consultaron artículos originales y de revisión en bases de datos: PubMed, CINAHL, SciELO, Medline, Lilacs, BIREME, Scival y Conhrane entre otras, según DeCS: Curriculum, Educación Basada en Competencias, Educación en Enfermería. El universo estudiado fue la totalidad de docentes de planta de la unidad académica, siendo abordable se estudió en totalidad $\mathrm{N}=21$. Los criterios de selección de participantes fueron: enfermeros docentes del staff de la carrera, con más de 5 años de permanencia en la misma. Resultados. El proceso de innovación curricular de la carrera de enfermería de la UTEQ, se encuentra inmerso en políticas y acuerdos que respondan al modelo educativo institucional; 
atendiendo al aumento de cobertura de la educación terciaria o superior, de transformaciones sociales y de cambios de paradigmas en salud. Con la innovación curricular, se buscó configurar un nuevo modelo de universidad que, sin descuidar la solvencia cognoscitiva y competencia profesional, ponga énfasis preferente en formación de sujetos reflexivos, responsables, críticos, innovadores y versátiles para garantizar cobertura universal de salud. Los principales procesos y estrategias que se usaron en esta innovación curricular estuvieron acorde a las políticas institucionales de las universidades, siguiendo lineamientos de los modelos educativos institucionales, objetivos estratégicos de docencia insertos en planes de desarrollo estratégico de las universidades.

Palabras claves: Modelo, integración, formación, profesional, enfermería

\section{Introducción}

Para esta innovación curricular, se siguieron bases y exigencias del modelo educativo institucional, requerimientos del Consejo de Aseguramiento de la Calidad de Educación Superior del Ecuador CACES, Secretaria de Educación Superior, Ciencia e Innovación SENESCYT, además de experiencias internacionales. Una primera fase (diseño) se ejecutó en base a cuatro Escuelas de Enfermería del país, seleccionadas según criterios de calidad y pertinencia. El inicio del proceso fue mediante metodología de taller, reuniones virtuales y presenciales, donde predominó el compromiso colaborativo entre los participantes, que tenían como objetivos revisar y estructurar la propuesta curricular en base a un plan de desarrollo estratégico de las universidades y políticas-procedimientos de los modelos educativos institucionales. También hubo pasantías internacionales para conocer realidades similares. El plan de estudio se estructura dando respuesta a marco de la cualificaciones y sistema de créditos transferibles, componente que convierte carga académica de estudiantes en créditos homologables entre universidades chilenas e introducción de actividades curriculares de formación básica, general y profesionalizantes, con contenidos y metodologías integradoras. Para ellos se declararon en el plan de estudio competencias genéricas y específicas a lograrse según cada nivel de formación del estudiante. Las competencias genéricas que declara la UTEQ como sello institucional son: liderazgo y emprendimiento, comunicación efectiva, usos de las Tics, trabajo colaborativo y en equipo. De este trabajo se obtiene un nuevo plan de estudio con un nuevo perfil de egreso; que da cuenta del compromiso formativo del estudiante, donde es posible diferenciar las dimensiones, así como, las competencias específicas y dominios que distinguen la formación en enfermería. Cada dominio se definió, en conjunto con expertos que guiaron las asesorías técnicas y académicos de enfermería. También se destacan en esta propuesta las competencias específicas por cada dominio, las cuales están en relación con cada uno de ellos y se aprecia el énfasis en el dominio disciplinar de enfermería en el ámbito de APS. Existe en esta innovación mayor proporción de horas de experiencia clínica en los entornos de 
atención primaria de salud, espacio donde el alumno materializan acciones promotoras y preventivas para alcanzar metas sanitarias y objetivos del milenio. Además, el rediseño busco integrar los principios y valores de la salud universal y la atención primaria de salud, además de los principios que sustentan las modalidades de educación transformadora, como el desarrollo del pensamiento crítico y complejo, la resolución de problemas, la toma de decisiones clínicas basadas en la evidencia y el aprendizaje a lo largo de toda la vida.

La Carrera de Enfermería de la Universidad Técnica Estatal de Quevedo UTEQ, institución educativa superior pública, guio su innovación por medio de su gestión basada en mejoramientos continuos, cambios necesarios del paradigma en la educación de las ciencias de la salud para responder a las necesidades de la población, asegurando la excelencia académica y vinculación con las comunidades que la acogen, reconociendo sus necesidades, contribuyendo al desarrollo integral y constante mejoramiento de su calidad de vida. Para ello, esta unidad académica se preocupa de la formación integral y continua de profesionales de enfermería con competencias genéricas y específicas de excelencia, señaladas en su perfil de egreso. En la innovación realizada queda establecido como las actividades curriculares responden a materializar progresión de competencias genéricas y específicas, idóneas para proveer y gestionar en el futuro profesional de enfermería con principios éticos, con visión y valores humanistas, sentido de responsabilidad y compromiso social, autonomía y liderazgo, respondiendo creativamente a la diversidad cultural y social en la que se desarrolle su acción, contribuyendo al fortalecimiento disciplinar y profesional por medio de la formación continua, en busca de la emancipación profesional.

La carrera, ha asumido como desafío, la concreción de un curriculum basado en competencias, desde una perspectiva integradora de saberes, situación que reconoce en su constante actualización del conocimiento, en la integración de ciencias básicas para nutrir la formación disciplinar y así romper la visión fragmentada de saberes, para situarse en fronteras interdisciplinarias, de atención de salud y así estar plenamente preparados para hacer los aportes máximos a la salud universal.

En este sentido, la innovación curricular también plantea un giro en la concepción del proceso formativo, en tanto, migra de un enfoque tradicional centrado en la enseñanza a uno centrado en el sujeto y su proceso de aprendizaje, reconociendo al estudiante como sujeto activo que construye conocimientos, responsable de su aprendizaje y repertorios de saberes, actitudes, habilidades de liderazgo, que condicionan una agencia cívica idónea para cambios significativos en el oscilante campo de la asistencia médica.

La tendencia es otras palabras, la convocatoria es atender a problemáticas de calidad y eficacia de los curriculum que tributan a grados y titulaciones, a la deserción de los estudiantes y mejor empleabilidad de egresados. Esta carrera ha optado por entrelazar un puente entre el enfoque curricular integrado y el enfoque curricular representado por la educación basada en competencias desde una perspectiva interdisciplinaria a fin de corregir 
el aprendizaje memorístico y abstracto para sustituirlo por actuaciones reflexivas, críticas, éticas y desempeños complejos.

Se materializan acciones a seguir en este proceso como: reuniones presenciales y virtuales entre académicos, pasantías internacionales, asesorías técnicas y trabajo grupal con resultados preliminares a corto y mediano plazo. Se suma a esto las revisiones frecuentes de literatura y las asesorías realizadas por los expertos internacionales en el ámbito curricular y disciplinar de Enfermería.

\section{Referencias Bibliográficas.}

Castillo M, Hawes G, Castillo S, Romero L, Rojas AM, Espinoza M. et al. Cambio educativo en las Facultades de Medicina. Rev. Méd. Chile. 2014:142(8)1056-1060. Disponible en http://www.scielo.cl/scielo.php?script=sci_arttext\&pid=S003498872014000800013\&lng=es\&nrm=isohttp://dx.doi.org/10.4067/S0034-

98872014000800013 [Fecha de consulta]: 9 ener. 2018. XVI Coloquio Panamericano de Investigación en Enfermería, Cuba 2018. Del 05 al 09 de Noviembre de 2018. La Habana, Cuba.

Cassiani SHDB, Wilson LL, Mikael SSE, Morán-Peña L, Zarate-Grajales R, McCreary LL, et al. The situation of nursing education in Latin America and the Caribbean towards universal health. Rev. Latino-Am. Enfermagem. 2017; 25:e2913. [access june, 04, 2020]; Available in: http://www.scielo.br/pdf/rlae/v25/es_0104-1169-rlae-25e2913.pdf. DOI: http://dx.doi.org/10.1590/1518-8345.2232.2913

Cerón, MC, A, "Impact of curricular change on the perception of the educational environment by nursing students". Invest Educ Enferm. 2015:33(1):63-72. Disponible en:

https://www.ncbi.nlm.nih.gov/pubmed/?term=Cer\%C3\%B3n\%20Mackay\%20MC\%5 BAuthor\%5D\&cauthor=true\&cauthor_uid=26148157 [Fecha de consulta]: 10 Julio 2020.

Clark KM , Miller JP, Leasing C, Baumgartner K. La enfermera ciudadana: una innovación educativa para el cambio. J Nurs Educ. 2017:56(4):247-250. Disponible en: https://www.ncbi.nlm.nih.gov/pubmed/28383752 doi:10.3928/01484834-2017032312 [Fecha de consulta]: 26 marzo 2020.

D'alfonso J, Jones D, Musgo T. Escuela de enfermería de Kaiser: un legado de 70 años de innovación disruptiva". Rev. Nurs Adm Q. 2018:42(1):35-42. Disponible en: https://www.ncbi.nlm.nih.gov/pubmed/29194331

doi:10.1097/NAQ.0000000000000262 [Fecha de consulta]: 9 junio 2020. 
Galarza López J, Soca González H, Almuiñas Rivero JL. Metodología para evaluar la calidad de la formación del estudiante en la Escuela Latinoamericana de Medicina. Educación Médica Superior [Internet]. 2018 [citado 2018 Abr 23];31(4):[aprox. 0 p.]. Disponible en: http://ems.sld.cu/index.php/ems/article/view/1131 XVI Coloquio Panamericano de Investigación en Enfermería, Cuba 2018. Del 05 al 09 de Noviembre de 2018. La Habana, Cuba.

Guzmán, A. Las competencias: otra mirada a la formación universitaria de la enfermería. Revista Electrónica Actualidades Investigativas en Educación. 2018:10(1):1-26. Disponible en: http://www.redalyc.org/articulo.oa?id=44713068016 [Fecha de consulta: 9 de enero de 2020.

Hernández, S. (2008). Metodología de la investigación. México: Interamericana. doi: 978607-15-0291-9

Hickey MT, Forbes M, Greenfield S. Integrar las competencias del instituto de medicina en una revisión curricular de bachillerato: procesos y estrategias". Rev. J Prof Nurs. 2010:26(4):214-22 Disponible en: https://www.ncbi.nlm.nih.gov/pubmed/20637443 doi:10.1016/j.profnurs.2010.03.001 [Fecha de consulta]: 05 julio. 2020.

Icarte GA, Labate HA. Metodología para la Revisión y Actualización de un Diseño Curricular de una Carrera Universitaria Incorporando Conceptos de Aprendizaje Basado en Competencias. Form. Univ. 2016 [acceso 09/01/202020];9(2):3-16. Disponible en http://www.scielo.cl/scielo.php?script=sci_arttext\&pid=S0718$50062016000200002 \& \operatorname{lng}=$ es\&nrm=iso

Ilkiw JE, Nelson RW, Watson JL, Conley AJ, Raybould HE, Chigerwe M, et al. Revisión y reforma curricular: el proceso, lo que era importante y las lecciones aprendidas. Rev. J Vet MedEduc.2017:44(3):480-489.Disponibleen: https://www.ncbi.nlm.nih.gov/pubmed/28876993 doi: 10.3138/jvme.0316-068R [Fecha de consulta]: 28 junio 2020.

Jensen GM, Nordstrom T, Mostrom E, Hack LM, Gwyer J. Estudio nacional de excelencia e innovación en educación física terapeuta. Diseño, método y resultados. Rev. Phys Tver. 2017:97(9):857-874. Disponible en: https://www.ncbi.nlm.nih.gov/pubmed/28586430 doy: 10.1093/paja/pzx061 [Fecha de consulta]: 10 ene. 2020.

LeDuc, K, Sedan T, Campbell L. Innovación curricular para integrar el arte y la ciencia de la enfermería. Journal of Nursing Education. 2013; 52(7):416. Disponible en: https://doi.org/10.3928/01484834-20130620-11 [Fecha de consulta]: 11 oct. 2017.

Landen J, Evans-Prior D, Dakin B, Liesveld J. Innovación en la progresión académica: progreso del modelo del consorcio de educación en enfermería de Nuevo México". 
Rev.

Nurs

EducPerspect.2017:38(5):E26-

E29.Disponibleen:https://www.ncbi.nlm.nih.gov/pubmed/28796134doi:10.1097/01.NE

P.0000000000000206 [Fecha de consulta]: 10 mayo 2020.

Mills, S, Krouse AM, Rossi-Schwartz R, Klein JM. Revisión del currículo: estrés del estudiante y lecciones aprendidas. Rev. J Nurs Educ, J Nurs Educ. 2017:56(6):337342. Disponible en: https://www.ncbi.nlm.nih.gov/pubmed/28585981 doi:10.3928/01484834-20170518-04. [Fecha de consulta]: 14 abril 2020.

Oliver BJ, Potter M, Pomerleau M, Phillips A, O'Donnell M, Cowley C, et al. Rápida integración de currículo de ciencia de mejora de la salud a través de programas en una escuela de enfermería”. Rev. Enfermera Educ. 2017:42 (5S Suppl 1):S38-S43. Disponible en: https://www.ncbi.nlm.nih.gov/pubmed/28832461 doi:10.1097/NNE.0000000000000428 [Fecha de consulta]: 15 marzo 2020.

Paulson C. Las experiencias de la docencia de la facultad en un innovador plan de estudios de enfermería de inmersión clínica". Rev. Nursing Education Perspectives, 2011:32(6):395399

Disponibleen:http://journals.lww.com/neponline/Abstract/2011/11000/THE_EXPERI ENCES_of_Fa culty_Teaching_in_an.11.aspx [Fecha de consulta]: 18 ener. 2020.

Vásquez, A.M. Innovación curricular en enfermería: Aprendizaje Integrado. Rev. Cuidado de Enfermería y Educación en Salud. 2014; 1(1):43-51. Disponible en: . [Fecha de consulta]: 16 mayo 2020. 


\section{PARA CITAR EL ARTÍCULO INDEXADO.}

Velázquez Lores, L. M., Mego Cubas, C., \& Pazmiño Solys, G. A. (2020). Modelo de integración de formación profesional de la carrera de licenciatura en enfermería en la UTEQ. ConcienciaDigital, 3(3.1), 160-167. https://doi.org/10.33262/concienciadigital.v3i3.1.1378

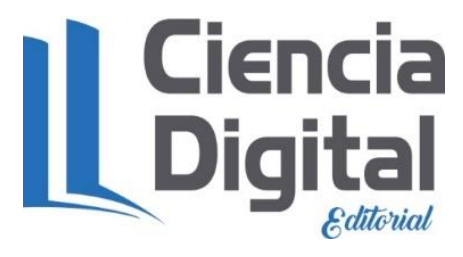

El artículo que se publica es de exclusiva responsabilidad de los autores y no necesariamente reflejan el pensamiento de la Revista Conciencia Digital.

El artículo queda en propiedad de la revista y, por tanto, su publicación parcial y/o total en otro medio tiene que ser autorizado por el director de la Revista Conciencia Digital.

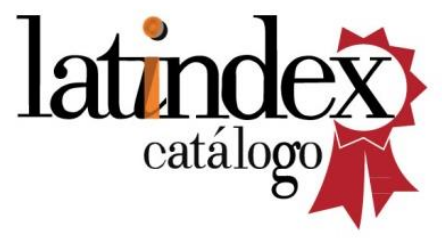

